\title{
The Relationship of Serum 25-Hydroxyvitamin-D Level with Severity of Obstructive Sleep Apnea in Patients with Type 2 Diabetes Mellitus
}

This article was published in the following Dove Press journal: Diabetes, Metabolic Syndrome and Obesity: Targets and Therapy

\begin{abstract}
Danyan Ma, ${ }^{1, *}$ Xuanling Zheng, ${ }^{2}$, * Lianqin Dong, ${ }^{3}$ Caiyu Zheng, Yun Chen, ${ }^{3}$ Zheng Chen, ${ }^{2}$ MingZhu Lin, ${ }^{2}$ Xuejun $\mathrm{Li}^{5}$ Zhibin Li, (iD) ${ }^{6}$ Changqin Liu ${ }^{3,7}$

'School of Medicine, Xiamen University, Xiamen City, Fujian Province, People's Republic of China;

${ }^{2}$ Department of Endocrinology and Diabetes, Xiamen Clinical Medical Center for Endocrine and Metabolic Diseases, Fujian Province Key Laboratory of Diabetes Translational Medicine, The First Affiliated Hospital of Xiamen University, Xiamen City, Fujian Province, People's Republic of China; ${ }^{3}$ The School of Clinical Medicine, Fujian Medical University, Fuzhou, Fujian Province, People's Republic of China; ${ }^{4}$ Fujian Medical University Xiamen Humanity Hospital, Xiamen City, Fujian Province, People's Republic of China;

${ }^{5}$ Department of Endocrinology and Diabetes, Xiamen Clinical Medical Center for Endocrine and Metabolic Diseases, Fujian Province Key Laboratory of Diabetes Translational Medicine, Xiamen Diabetes Institute, The First Affiliated Hospital of Xiamen University, Xiamen City, Fujian Province, People's Republic of China; ${ }^{6}$ Epidemiology Research Unit, The First Affiliated Hospital of Xiamen University, Xiamen City, Fujian Province, People's Republic of China;

${ }^{7}$ Department of Endocrinology and Diabetes, Xiamen Clinical Medical Center for Endocrine and Metabolic Diseases, Fujian Province Key Laboratory of Diabetes Translational Medicine, The First Affiliated Hospital of Xiamen University, Xiamen City, Fujian Province. People's Republic of China
\end{abstract}

*These authors contributed equally to this work

Correspondence: Changqin Liu

Department of Endocrinology and Diabetes, Xiamen Clinical Medical Center for Endocrine and Metabolic Diseases, Fujian Province Key Laboratory of Diabetes Translational Medicine, The First Affliated Hospital of Xiamen University, Xiamen City, Fujian Province,

People's Republic of China

Tel +86-592-2137610

Fax +86-592-2137557

Email liuchangqin@xmu.edu.cn

Zhibin Li

Epidemiology Research Unit, The First Affiliated Hospital of Xiamen University, Xiamen City, Fujian Province, People's Republic of China

Tel +86-592-2137364

Fax +86-592-2137557

Email zhibinli33@hotmail.com
Objective: To explore the association of serum vitamin-D levels with the severity of obstructive sleep apnea (OSA) in patients with type 2 diabetes mellitus (T2DM).

Methods: A cross-sectional study of 136 patients with T2DM who underwent overnight polysomnography (PSG) tests and serum 25-hydroxyvitamin-D3 (25(OH)D3) level detections was conducted. Multivariable linear regression and logistic regression analyses were performed to determine the associations of serum 25(OH)D3 levels with apnea-hypopnea index (AHI) and obstructive sleep apnea (OSA).

Results: The prevalence rates of OSA were $84.4 \%$ for male and $65.2 \%$ for female patients, respectively ( $p=0.011$ ). With increasing severities of OSA categories (none, mild, moderate and severe), patients were more likely to be male and obese, but there was no significant difference in serum 25(OH)D3 level ((mean \pm standard deviation) $21.8 \pm 8.8,27.7 \pm 14.6$, $24.2 \pm 9.8$ and $26.8 \pm 6.2 \mathrm{ng} / \mathrm{mL}$, respectively, $\mathrm{p}=0.086)$. Serum 25(OH)D3 level was not significantly correlated with AHI (log-transformed), with the correlation coefficient of 0.133 $(\mathrm{p}=0.124)$. With adjustment for potential confounding factors, multivariable linear regression and logistic regression analyses showed that serum 25(OH)D3 level was not significantly associated with either AHI (log-transformed) or the risk of OSA, with the standardized regression coefficient (95\% confidence interval (CI)) of $0.098(-0.004-0.014, \mathrm{p}=0.252)$ and the adjusted odds ratio $(95 \% \mathrm{CI})$ of $1.055(0.991-1.124, \mathrm{p}=0.095)$, respectively.

Conclusion: Serum 25(OH)D3 level was not significantly associated with either AHI or the risk of OSA in patients with T2DM.

Keywords: obstructive sleep apnea, polysomnography, 25-hydroxyvitamin-D3, apneahypopnea index, type 2 diabetes mellitus

\section{Introduction}

Obstructive sleep apnea (OSA) is a widespread clinical syndrome characterized by the snoring and sudden apnea during sleep resulting in intermittent hypoxia which is caused by recurrent, partial or complete upper airway obstruction. ${ }^{1}$ The prevalence rate of OSA is $10 \sim 17 \%$ among men and 3 9\% among women in developed countries. $^{2}$ Usually, OSA develops in sub-populations with overweight and obesity and is associated with hypertension and metabolism-related diseases. ${ }^{3,4}$ Previous studies indicated that OSA was more common (prevalence rate is from $50 \%$ to $70 \%$ ) in type 2 diabetes (T2DM). ${ }^{5}$ T2DM may be a risk factor of OSA explained by the mechanism of inflammation and oxidative stress. ${ }^{6}$ In turn, intermittent hypoxia caused by OSA leads to endocrine disorder, such as insulin resistance (IR), 
impaired glucose tolerance and development of T2DM. ${ }^{7}$ Aronsohn et $\mathrm{al}^{8}$ showed that glycaemic control would get worse in T2DM patients with the progression of OSA, as measured by their glycated hemoglobin (HbAlc) levels after adjusting for age, race, sex, body mass index and number of years with DM.

It is well known that vitamin D plays an important role in calcium homeostasis and bone metabolism, which is acquired from dietary source and skin exposure to solar radiation. ${ }^{9}$ Vitamin D deficiency is defined as the level lower than 20 $\mathrm{ng} / \mathrm{mL}$, and vitamin D sufficiency is defined as the vitamin D concentration higher than $30 \mathrm{ng} / \mathrm{mL} .{ }^{10}$ It is known that Vitamin D insufficiency $(21-30 \mathrm{ng} / \mathrm{mL})$ is a common condition and the consequences of the increasing prevalence of vitamin D insufficiency are not well studied. In addition, there are racial or religious differences in vitamin D levels. ${ }^{11}$ Available evidence suggests that vitamin D deficiency would lead to the increasing of prevalence and severity of metabolic disorders, obesity, cardiovascular diseases and respiratory tract infections. ${ }^{12-15}$ Research regarding the association between vitamin D and severity of OSA has become a hot spot but the results are controversial. Some meta-analyses have reported that patients with OSA have lower vitamin D levels than healthy patients; ${ }^{16}$ and others have shown that vitamin D deficiency is more common in those with severe OSA. ${ }^{17}$ Of interest, vitamin D insufficiency shares mutual risk factors with OSA, such as age, obesity, hypertension, kidney diseases and diabetes. ${ }^{18,19}$ Nevertheless, among individuals with T2DM, the relationship between vitamin D level and severity of OSA remains unclear.

To fill this knowledge gap, the present study aimed to explore the association of serum vitamin $\mathrm{D}$ levels with severity of OSA in patients with type 2 diabetes, which would determine that whether screening serum vitamin D concentration is necessary for assessing Vitamin D insufficiency or even deficiency in diabatic patients with OSA.

\section{Methods}

\section{Ethics Statement}

The study was approved by the Human Research Ethics Committee of the Xiamen First Hospital of Xiamen University (Xiamen, China). Written informed consent was obtained from each participant.

\section{Study Population}

A total of 150 patients with T2DM from the Department of Endocrinology and Diabetes, the First Affiliated Hospital of Xiamen University (Xiamen, China) had been recruited into the present cohort. Patients with T2DM with symptoms indicating sleep-related breathing disorders were invited to participate in this study. Exclusion criteria were as follows: the presence of hyperthyroidism or hypothyroidism, acute illnesses, serious heart diseases, chronic kidney disease, uncontrolled hypertension, presence of cancer, craniofacial abnormalities, any respiratory disorder other than OSA, and current use of hypnotics or any treatment for breathing disorders. Face-to-face interview was conducted for each patient to collect basic information, tobacco smoking, alcohol consumption, medical history, and sleep state. Besides, all patients admitted routine check-up evaluations, detailed physical examination and overnight polysomnography (PSG). Of them, 136 patients who had complete data on clinical, biochemical and PSG measurements were left for the present analysis.

\section{Anthropometric and Biochemical Measurements}

Anthropometric measurements include body weight, height, waist circumference (WC), blood pressure (BP), and body mass index (BMI). WC was measured at the midpoint between the inferior costal margin and the superior border of the iliac crest on the mid-axillary line. Body weight and height were measured by using a calibrated scale after removing shoes and heavy clothes to the nearest $0.1 \mathrm{~kg}$ and $0.5 \mathrm{~cm}$, respectively. BMI was calculated as the weight in kilograms divided by the square of the height in meters. $\mathrm{BP}$ was recorded using an electronic sphygmomanometer as the average of three consecutive measurements separated by a 1-min interval after sitting for at least 15 minutes.

All blood samples were obtained after 12-hour fasting. Blood samples were tested in the central laboratory of the First Affiliated Hospital of Xiamen University. Triglyceride (TG), total cholesterol (TC) and high-density lipoprotein cholesterol (HDL-c) were determined on a HITACHI 7450 analyzer (HITACHI, Tokyo, Japan). Low-density lipoprotein cholesterol (LDL-c) was calculated by Friedewald's formula. Hemoglobin A1c (HbAlc) was measured by the Bio-Rad VARIANT HbAlc assay. And serum 25-hydroxyvitamin-D3 (25(OH)D3) levels were measured using a Roche Cobas E411 autoanalyzer (Roche, Germany) with an electrochemiluminescence immunoassay (Measuring range: $3.00-70.0 \mathrm{ng} / \mathrm{mL}$ ). Seasons of blood sampling were recorded for all patients. As this study was conducted in Xiamen (latitude $24.48^{\circ} \mathrm{S}$ ), which is a hot and highly sunny city in south of China, the summer- 
autumn season was defined as from May to October and the spring-winter season was from November to April next year.

\section{Polysomnography}

PSG was performed using a digital polygraph on each patient (Alice PDX, Philips Respironics, USA) from 11:00 pm to 7:00 am in the hospital. And the electroencephalogram (EEG), electrooculogram (EOG), electromyogram (EMG), flow, thoracic and abdominal respiratory effort, oximetry, and body position were monitored. Polysomnographic recordings were interpreted in accordance with the American Academy of Sleep Medicine (AASM) Manual for the Scoring of Sleep and Associated Events. ${ }^{20}$ The recording duration $\geq 5 \mathrm{~h}$ was required for validation, and monitoring was repeated on a second night if subjective sleep latency exceeded $2 \mathrm{~h}$ on the first night or if respiratory parameters were missing. The apnea-hypopnea index (AHI) was defined as the total number of obstructive apnea and hypopnea per hour of sleep, and the severity of OSA was determined by AHI. None, mild, moderate, and severe OSA were defined by an $\mathrm{AHI} \leq 4.9,5-14.9,15$ 29.9 , and $\geq 30$ events/hour, respectively. ${ }^{21}$

\section{Statistical Analysis}

Data was presented as the mean \pm standard deviation or as median (inter-quartile range, IQR) for continuous variable or number and percentage for the categorical variable. Skewness and kurtosis tests for normality of serum 25(OH)D3 level and AHI were conducted and found serum 25(OH)D3 but not AHI followed normal distribution. Differences between subjects (categorized by severity of OSA: none, mile, moderate and severe) were analyzed on continuous variables using one-way ANOVA for those with normal distribution and KruskalWallis test for those with skewed distribution and on categorical variables using chi-square test. As AHI did not follow the normal distribution, AHI was log-transformed in the following correlation and regression analyses. The correlation of serum 25(OH)D3 level with AHI (log-transformed) was analyzed using Pearson's correlation analysis. Multivariable linear regression was used to explore the associations of serum 25 $(\mathrm{OH}) \mathrm{D} 3$ level with AHI (log-transformed). And multivariable logistic regression analysis was used to calculate the adjusted odds ratios (OR) and 95\% confidence interval (CI) of serum 25(OH)D3 level for OSA in different models with adjustment for potential confounders. For both the multivariable linear regression and logistic regression analyses, no variables were adjusted for in model 1; age, sex, regular drinking, current smoking, T2DM duration, systolic blood pressure (SBP) and diastolic blood pressure (DBP) were adjusted for in model 2, and BMI, TC, TG, HbAlc, seasons of blood sampling were further adjusted for in model 3. All p-values presented are twotailed, and values less than 0.05 are considered statistically significant. All statistical analyses were performed using IBM SPSS Statistics 21.0.

\section{Results}

Totally, there were 136 patients with T2DM left for the present analyses. Among them, 90 (66.2\%) were men, and the mean ages $( \pm \mathrm{SD})$ were $47.3( \pm 13.2)$ years for men and $52.2( \pm 15.2)$ years for women $(\mathrm{p}=0.058)$. The total prevalence rates of OSA were $84.4 \%$ for men and $65.2 \%$ for women, respectively $(\mathrm{p}=0.011)$.

\section{Clinical Characteristics of Patients Stratified by Severity of OSA}

The overall prevalence rate of OSA was $77.9 \%$ for all patients. In terms of severity of OSA, the prevalence rates were $24.3 \%$, $22.8 \%$ and $30.8 \%$ for mild, moderate and severe OSA, respectively. Table 1 shows the differences of demographics, lifestyle habits and clinical characteristics stratified by severities of OSA (none, mild, moderate and severe OSA). There were no significant differences among these four groups in age (43.6 \pm 13.8 , $53.2 \pm 15.2,48.8 \pm 13.5,49.7 \pm 12.7$, respectively, $\mathrm{p}=0.054)$, regular drinking, current smoking, duration of T2DM, SBP and DBP, triglyceride, total cholesterol, HDL-c, LDL-c, HbA1c, seasons of blood sampling, and serum 25(OH)D3 level $(25.3$ $\pm 10.3,21.8 \pm 8.8,27.7 \pm 14.6,24.2 \pm 9.8,26.8 \pm 6.2$, respectively, $\mathrm{p}=0.086$ ). However, there were significant differences in gender, WC $(94.5 \pm 10.3,97.8 \pm 8.7,100.0 \pm 11.4,102.9 \pm 12.3$, respectively, $\mathrm{p}=0.013)$, BMI $(27.7 \pm 3.4,27.0 \pm 3.5,28.8 \pm 5.3$, $30.1 \pm 4.5$, respectively, $\mathrm{p}=0.012)$ and AHI (2.9 (1.2-4.3), 8.8 (5.8-12.1), 20.7 (18.3-26.3), 44.1 (38.4-58.2) events/hour, respectively, $\mathrm{p}<0.001$ ) among these four groups.

\section{Correlation of Serum 25(OH)D3 Level with $\mathrm{AHI}$}

Figure 1 shows there was no significant correlation of serum 25(OH)D3 level with AHI (log-transformed), with the correlation coefficient of $0.133(\mathrm{p}=0.124)$. Figure 2 shows the difference of AHI stratified by tertiles of serum 25(OH)D3 level, and it shows there was a significant difference between tertiles 1 and tertiles 3, with AHI median (IQR) of 6.0 (18.8) and 27.1 (31.7), respectively $(\mathrm{p}=0.002)$. However, there was no significant difference of AHI between tertile 1 and tertile 2 or between tertile 2 and tertile $3(\mathrm{p}=0.187$ and $\mathrm{p}=0.412$, respectively). 
Table I Demographic, Lifestyle and Clinical Characteristics of Study Subjects by Severities of OSA

\begin{tabular}{|c|c|c|c|c|c|c|}
\hline & \multirow[b]{2}{*}{ Total } & \multicolumn{4}{|c|}{ Severity of OSA } & \multirow[b]{2}{*}{$\mathbf{p}$} \\
\hline & & None & Mild & Moderate & Severe & \\
\hline N (\%) & 136 & $30(22.1 \%)$ & $33(24.3 \%)$ & $3 \mathrm{I}(22.8 \%)$ & $42(30.8 \%)$ & \\
\hline Age (years) & $49.0 \pm 14.0$ & $43.6 \pm 13.8$ & $53.2 \pm 15.2$ & $48.8 \pm 13.5$ & $49.7 \pm 12.7$ & 0.054 \\
\hline Gender & & & & & & 0.046 \\
\hline Female (n, \%) & $46(33.8 \%)$ & $16(53.3 \%)$ & II (33.3\%) & $10(32.3 \%)$ & 9 (21.4\%) & \\
\hline Male (n, \%) & $90(66.2 \%)$ & 14 (46.7\%) & $22(66.7 \%)$ & 21 (67.7\%) & $33(78.6 \%)$ & \\
\hline Regular drinking (\%) & 43 (31.6\%) & $8(26.7 \%)$ & $9(27.3 \%)$ & $12(38.7 \%)$ & $14(33.3 \%)$ & 0.704 \\
\hline Current smoking (\%) & 54 (39.7\%) & 8 (26.7\%) & $13(39.4 \%)$ & $16(51.6 \%)$ & $17(40.5 \%)$ & 0.264 \\
\hline T2DM duration (years) & $4.0(1.0-9.0)$ & $3.5(0.8-8.5)$ & $5.0(1.5-11.0)$ & $2.0(1.0-7.0)$ & $4.5(1.8-9.0)$ & 0.619 \\
\hline$W C(\mathrm{~cm})$ & $99.2 \pm 11.2$ & $94.5 \pm 10.3$ & $97.8 \pm 8.7$ & $100.0 \pm 11.4$ & $102.9 \pm 12.3$ & 0.013 \\
\hline BMI $\left(\mathrm{kg} / \mathrm{m}^{2}\right)$ & $28.6 \pm 4.4$ & $27.7 \pm 3.4$ & $27.0 \pm 3.5$ & $28.8 \pm 5.3$ & $30.1 \pm 4.5$ & 0.012 \\
\hline $\mathrm{SBP}(\mathrm{mmHg})$ & $131.6 \pm 16.5$ & $132.6 \pm 15.4$ & $128.8 \pm 15.7$ & $133.9 \pm 15.5$ & $131.4 \pm 18.6$ & 0.660 \\
\hline $\mathrm{DBP}(\mathrm{mmHg})$ & $82.2 \pm 9.8$ & $82.8 \pm 10.6$ & $78.3 \pm 10.0$ & $84.1 \pm 8.5$ & $83.4 \pm 9.5$ & 0.069 \\
\hline Triglyceride (mmol/L) & $1.6(1.2-2.6)$ & I.4 (I.I-2.9) & $1.6(1.3-2.2)$ & $1.6(1.2-2.9)$ & $1.8(1.3-3.7)$ & 0.509 \\
\hline $\mathrm{TC}(\mathrm{mmol} / \mathrm{L})$ & $5.1 \pm 1.3$ & $4.9 \pm 1.3$ & $4.8 \pm 1.0$ & $5.1 \pm 1.6$ & $5.4 \pm 1.3$ & 0.191 \\
\hline HDL-c (mmol/L) & $1.2 \pm 0.4$ & $1.2 \pm 0.7$ & $\mathrm{I} . \mathrm{I} \pm 0.3$ & $1.2 \pm 0.4$ & $1.2 \pm 0.3$ & 0.508 \\
\hline LDL-c (mmol/L) & $2.9 \pm 0.9$ & $2.7 \pm 1.1$ & $2.9 \pm 0.8$ & $2.8 \pm 0.8$ & $3.0 \pm 1.0$ & 0.481 \\
\hline HbAlc (\%) & $9.6 \pm 2.0$ & $9.3 \pm 1.9$ & $9.2 \pm 1.8$ & $10.2 \pm 2.1$ & $9.5 \pm 2.2$ & 0.191 \\
\hline AHI (events/h) & $17.8(5.2-37.2)$ & $2.9(1.2-4.3)$ & $8.8(5.8-12.1)$ & $20.7(18.3-26.3)$ & $44.1(38.4-58.2)$ & $<0.001$ \\
\hline Season of blood sampling & & & & & & 0.156 \\
\hline Spring-winter season & 57 (4I.9\%) & $17(56.7 \%)$ & $10(30.3 \%)$ & II (35.5\%) & $19(45.2 \%)$ & \\
\hline Summer-autumn season & 79 (58.1\%) & $13(43.8 \%)$ & $23(69.7 \%)$ & $20(64.5 \%)$ & $23(54.8 \%)$ & \\
\hline Serum 25(OH)D3 level (ng/mL) & $25.3 \pm 10.3$ & $21.8 \pm 8.8$ & $27.7 \pm 14.6$ & $24.2 \pm 9.8$ & $26.8 \pm 6.2$ & 0.086 \\
\hline
\end{tabular}

Notes: Values are mean \pm SD, median (inter-quartile range) or percentage (column percentage).

Abbreviations: WC, waist circumference; BMI, body mass index; SBP, systolic blood pressure; DBP, diastolic blood pressure; TC, total cholesterol; HDL-c, high-density lipoprotein cholesterol; LDL-c, low-density lipoprotein cholesterol; HbAlc, hemoglobin Alc; T2DM, type 2 diabetes mellitus; AHI, apnea-hypopnea index; OSA, obstructive sleep apnea; 25(OH)D3, 25-hydroxyvitamin-D3.

\section{Association of Serum 25(OH)D3 Level with AHI}

Multivariable linear regression analyses were performed in order to exhibit the association of serum 25(OH)D3 level with AHI (log-transformed) (Table 2). In model 1 without

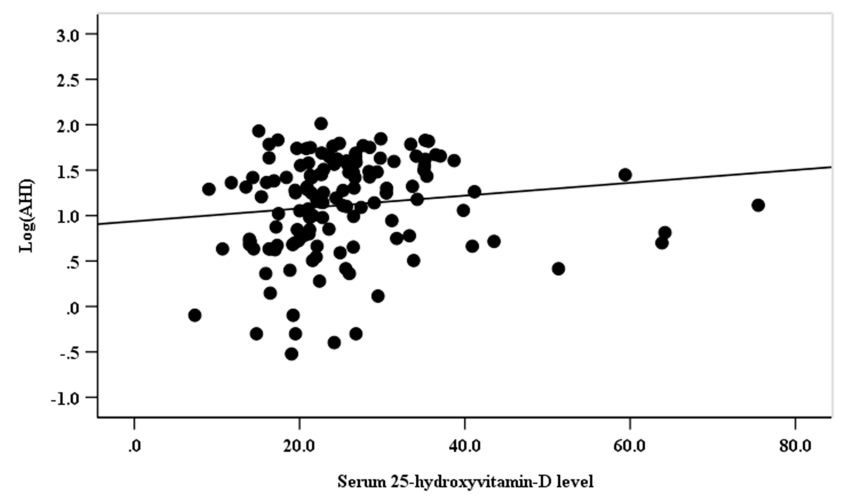

Figure I Correlation of serum 25-hydroxyvitamin-D level with log-transformed apnea-hypopnea index (AHI) in all subjects. adjustment for any confounding factor, serum 25(OH)D3 level was not significantly associated with AHI, and the standardized regression coefficient $(95 \% \mathrm{CI})$ was 0.133 $(-0.037-0.302, \mathrm{p}=0.124)$. In model 2 (with adjustment

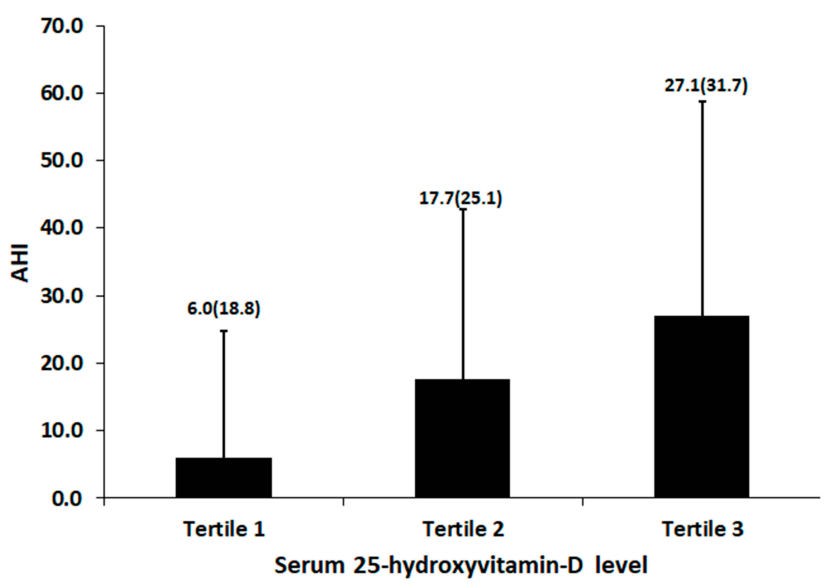

Figure 2 Apnea-hypopnea index (AHI) stratified by tertiles of serum 25hydroxyvitamin-D level in all subjects. 
Table 2 Association of Serum 25(OH)D3 Level with OSA

\begin{tabular}{|l|l|l|l|l|l|l|}
\hline & \multicolumn{2}{l}{ Linear Regression on AHI } & \multicolumn{2}{l|}{ Logistic Regression on OSA } \\
\cline { 2 - 7 } & Coefficient & $\mathbf{9 5 \%} \mathbf{~ I ~}$ & P value & OR & $\mathbf{9 5 \%}$ CI & P value \\
\hline $\begin{array}{l}\text { Model I } \\
\text { Serum 25(OH)D3 level }\end{array}$ & 0.133 & $-0.037-0.302$ & 0.124 & 1.065 & $1.005-1.129$ & $0.033 *$ \\
\hline $\begin{array}{l}\text { Model 2 } \\
\text { Serum 25(OH)D3 level }\end{array}$ & 0.074 & $-0.098-0.246$ & 0.394 & 1.050 & $0.989-1.114$ & 0.111 \\
\hline $\begin{array}{l}\text { Model 3 } \\
\text { Serum 25(OH)D3 level }\end{array}$ & 0.098 & $-0.070-0.266$ & 0.252 & 1.055 & $0.991-1.124$ & 0.095 \\
\hline
\end{tabular}

Notes: ${ }^{\mathrm{p}} \mathrm{p}<0.05$, AHI was log-transformed. Model I was not adjusted. Model 2 was adjusted for age, gender, T2DM duration, regular drinking, current smoking. Model 3 was further adjusted for SBP, DBP, BMI, TC, LDL-c, HbAlc.

Abbreviations: T2DM, type 2 diabetes mellitus; SBP, systolic blood pressure; DBP, diastolic blood pressure; BMI, body mass index; TC, total cholesterol; LDL-c, lowdensity lipoprotein cholesterol; HbAlc, hemoglobin Alc; AHI, apnea-hypopnea index; OSA, obstructive sleep apnea; 25(OH)D3, 25-hydroxyvitamin-D3.

for age, gender, duration of T2DM, regular drinking, current smoking, SBP and DBP) and model 3 (with further adjustment for BMI, TC, triglyceride, $\mathrm{HbA} 1 \mathrm{c}$, and season of blood sampling), serum 25(OH)D3 level was still not significantly associated with AHI, and the standardized regression coefficients $(95 \% \mathrm{CI})$ were $0.074(-0.098-$ $0.246, \mathrm{p}=0.394)$ and $0.098 \quad(-0.070-0.266, \mathrm{p}=0.252)$, respectively.

\section{Association of Serum 25(OH)D3 Level with OSA}

Multivariable logistic regression analyses were performed to show the association of serum $25(\mathrm{OH}) \mathrm{D} 3$ level with OSA (Table 2). In model 1 without adjustment for any confounding factor, increased serum $25(\mathrm{OH}) \mathrm{D} 3$ level was significantly associated with increased risk of OSA, with the adjusted OR (95\% CI) of 1.065 (1.005-1.129, $\mathrm{p}=0.033$ ). However, in model 2 and model 3 with the same adjustments as those in multivariable linear regression analyses, serum 25(OH)D3 level was not significantly associated with risk of OSA, with the adjusted OR $(95 \%$ CI) of $1.050(0.989-1.114, \mathrm{p}=0.111)$ and $1.055(0.991--$ $1.124, \mathrm{p}=0.095)$, respectively.

\section{Discussion}

In the present study, we have shown that there was no significant difference in serum $25(\mathrm{OH}) \mathrm{D} 3$ level with increasing severities of OSA (none, mild, moderate and severe OSA) and that serum $25(\mathrm{OH}) \mathrm{D} 3$ level was not significantly correlated with AHI. After adjusting for potential confounding factors in both multivariable linear regression and logistic regression analyses, we found that serum 25(OH)D3 level was not significantly associated with either AHI or OSA.

It has been established that vitamin $\mathrm{D}$ has extensive effects and plays a key role in an extended range of organs' functions and disorders, such as bone metabolism and calcium homeostasis. ${ }^{22}$ And it is well-known that abnormality of vitamin D would lead to osteoporosis, obesity, hypertension, malignant tumor, all kinds of infections and metabolic disorders. ${ }^{23}$ Vitamin D deficiency and OSA share the same risk factors and comorbidities, particularly older age, obesity, renal failure and diabetes. However, studies on the relationship between serum vitamin D level and OSA severity expressed by AHI have contradictory results. Some studies reported that patients with OSA have lower vitamin D levels than those without. In a cross-sectional study recruited by general population conducted in Ireland, ${ }^{17}$ the authors found that there were significant, independent inverse relationships between serum 25(OH)D3 levels and AHI, which is consistent with the two observational studies with few sample sizes. $^{22,24}$ As in OSA patients with metabolic syndrome, ${ }^{25}$ serum 25(OH)D3 levels were significantly decreased. Patients with OSA and insulin resistance (IR) had lower levels of serum $25(\mathrm{OH}) \mathrm{D} 3$ compared with those without IR, further regression analysis found a negative association of $25(\mathrm{OH}) \mathrm{D} 3$ levels with IR. ${ }^{26,27}$ One possible explanation for the above findings maybe that vitamin D deficiency enhanced IR by promoting inflammation. ${ }^{28}$ Additionally, vitamin D may contribute to the increased risks of infections and inflammations which are involved in the upper and lower airway. ${ }^{29}$ However, there were also some studies reporting the opposite findings. Mete et al found that there was no significant difference regarding the vitamin D levels between OSA patients and the controls, although the vitamin D levels 
were lower in severe patients than those in mild and moderate groups. ${ }^{30}$ Yassa et al found no significant difference in the vitamin-D levels within the OSA group and no significant relationship between vitamin-D and AHI. ${ }^{31}$ Similarly, another cross-sectional study conducted in Turkey, ${ }^{9}$ the researchers could not find a significant association of vitamin D levels with AHI or BMI. In a multicenter cohort study with 2827 community-dwelling, largely white, elderly males, Goswami et $\mathrm{al}^{32}$ observed that the lowest quartile of 25 (OH)D3 had greater odds of severe OSA compared to the highest 25(OH)D3 quartile in unadjusted analyses, but after adjusting for BMI and neck circumference as the main confounders there was no significant association between the 25 $(\mathrm{OH}) \mathrm{D} 3$ levels and increased risk of OSA.

Many previous studies have shown vitamin $\mathrm{D}$ deficiency is more common in patients with T2DM, regardless of age, sex, or insulin treatment. ${ }^{33}$ Some longitudinal and observational studies have shown that low levels of vitamin D predict T2DM risks in Europeans, ${ }^{34}$ African-Americans, ${ }^{35}$ South Asians ${ }^{36}$ and native American children. ${ }^{37} \mathrm{~A}$ study investigating the relationship between vitamin D and T2DM points out that vitamin D supplements are effective in decreasing the risk of $\mathrm{T}^{\mathrm{DDM}}{ }^{38}$ Song et al reported a $38 \%$ lower risk of developing T2DM in the highest category of vitamin D versus the lowest one in a meta-analysis of prospective studies. ${ }^{39}$ In an early cross-sectional study recruiting 91 non-diabetic subjects, 75 prediabetics and 24 diabetics, the patients with prediabetes or diabetes tended to present lower vitamin D level. ${ }^{40}$ However, there were scarce studies to explore the association between serum vitamin D level and OSA in T2DM patients. In the present study, we found there was no significant difference in serum 25(OH)D3 level with increasing severities of OSA and that there was no significant association of serum 25(OH)D3 level with either AHI or OSA in patients with T2DM. The first possible explanation was that all the participants in the current study were T2DM patients and had relatively lower levels of vitamin D than general subjects. Of our $136 \mathrm{~T} 2 \mathrm{DM}$ patients, $68(50 \%)$ were classified as vitamin D insufficient (21-30 ng/ $\mathrm{mL})$ and $39(28.7 \%)$ as deficient $(</=20 \mathrm{ng} / \mathrm{mL})$. The second possible reason was we did not have data on information on vitamin D supplement treatment and accurate sunshine exposure time for each participant although we have adjusted for the seasonality as one potential confounding factor.

This study has some limitations. The first major limitation was that most of our T2DM patients were obese with a relatively higher prevalence of OSA. Around $78 \%$ of our subjects were OSA and we may, therefore, under-estimate the true associations of serum 25(OH)D3 level with AHI and OSA. Whether our findings could be extrapolated to other T2DM patients is not determined. Another major limitation was that the number of subjects was small and we might not have sufficient power. Therefore, larger study, especially from a prospective cohort study design, is essential to assess the true relation of vitamin D with OSA in T2DM patients. Third, the present study was designed as a cross-sectional study; therefore, we cannot preclude the possibility of reverse causality between serum 25(OH)D3 and OSA. Finally, it also important to investigate the effect of vitamin D on the quality of life of T2DM patients with OSA in the future study.

\section{Conclusion}

To sum up, there was no significant difference in serum 25 $(\mathrm{OH}) \mathrm{D}$ level with increasing severities of OSA; and serum 25(OH)D3 level was not significantly associated with either AHI or OSA. However, further investigation is needed to clarify the relationship between vitamin $\mathrm{D}$ and OSA in patients with T2DM.

\section{Ethical Approval}

All procedures performed in studies involving human participants were in accordance with the ethical standards of the institutional and/or national research committee and with the Helsinki Declaration and its later amendments or comparable ethical standards.

\section{Acknowledgments}

We are grateful to all the patients for their participation.

\section{Author Contributions}

All authors made substantial contributions to conception and design, acquisition of data, or analysis and interpretation of data; took part in drafting the article or revising it critically for important intellectual content; gave final approval of the version to be published; and agree to be accountable for all aspects of the work.

\section{Funding}

CL was funded by the Natural Science Foundation of China grant no: 81870611. ML was funded by the Natural Science Foundation of Fujian Province grant no: 2017J01365.

\section{Disclosure}

The authors declare that they have no conflict of interest. 


\section{References}

1. Veasey SC, Rosen IM, Solomon CG. Obstructive sleep apnea in adults. $N$ Engl $J$ Med. 2019;380(15):1442-1449. doi:10.1056/ NEJMcp 1816152

2. Peppard PE, Young T, Barnet JH, Palta M, Hagen EW, Hla KM. Increased prevalence of sleep-disordered breathing in adults. $\mathrm{Am}$ J Epidemiol. 2013;177:1006-1014. doi:10.1093/aje/kws342

3. Redline S, Tishler PV, Schluchter M, Aylor J, Clark K, Graham G. Risk factors for sleep-disordered breathing in children. Associations with obesity, race, and respiratory problems. Am J Respir Crit Care Med. 1999;159:1527-1532. doi:10.1164/ajrccm.159.5.9809079

4. Lombardi C, Tobaldini E, Montano N, Losurdo A, Parati G. Obstructive Sleep Apnea Syndrome (OSAS) and cardiovascular system. Med Lav. 2017;108:276-282. doi:10.23749/mdl.v108i4.6427

5. Einhorn D, Stewart DA, Erman MK, Gordon N, Philis-Tsimikas A, Casal E. Prevalence of sleep apnea in a population of adults with type 2 diabetes mellitus. Endocr Pract. 2007;13:355-362. doi:10.4158/ EP.13.4.355

6. Shen H, Zhao J, Liu Y, Sun G. Interactions between and shared molecular mechanisms of diabetic peripheral neuropathy and obstructive sleep apnea in type 2 diabetes patients. J Diabetes Res. 2018;2018:3458615. doi:10.1155/2018/3458615

7. Nagayoshi M, Punjabi NM, Selvin E, et al. Obstructive sleep apnea and incident type 2 diabetes. Sleep Med. 2016;25:156-161. doi:10.1016/j.sleep.2016.05.009

8. Aronsohn RS, Whitmore H, Van Cauter E, Tasali E. Impact of untreated obstructive sleep apnea on glucose control in type 2 diabetes. Am J Respir Crit Care Med. 2010;181:507-513. doi:10.1164/rccm.200909-14230C

9. Salepci B, Caglayan B, Nahid P, et al. Vitamin D deficiency in patients referred for evaluation of obstructive sleep apnea. $J$ Clin Sleep Med. 2017;13:607-612. doi:10.5664/jcsm.6554

10. Holick MF. Vitamin D deficiency. N Engl J Med. 2007;357:266-281. doi:10.1056/NEJMra070553

11. Hilger J, Friedel A, Herr R, et al. A systematic review of vitamin D status in populations worldwide. Br J Nutr. 2014;111:23-45. doi: $10.1017 /$ S0007114513001840

12. Pacifico L, Anania C, Osborn JF, et al. Low 25(OH)D3 levels are associated with total adiposity, metabolic syndrome, and hypertension in Caucasian children and adolescents. Eur $J$ Endocrinol. 2011;165:603-611. doi:10.1530/EJE-11-0545

13. Olson ML, Maalouf NM, Oden JD, White PC, Hutchison MR. Vitamin D deficiency in obese children and its relationship to glucose homeostasis. J Clin Endocrinol Metab. 2012;97:279-285. doi:10.1210/jc.2011-1507

14. Zhang R, Li B, Gao X, et al. Serum 25-hydroxyvitamin D and the risk of cardiovascular disease: dose-response meta-analysis of prospective studies. Am J Clin Nutr. 2017;105:810-819. doi:10.3945/ ajen.116.140392

15. Science M, Maguire JL, Russell ML, Smieja M, Walter SD, Loeb M. Low serum 25-hydroxyvitamin D level and risk of upper respiratory tract infection in children and adolescents. Clin Infect Dis. 2013;57:392-397. doi:10.1093/cid/cit289

16. Neighbors CLP, Noller MW, Song SA, et al. Vitamin D and obstructive sleep apnea: a systematic review and meta-analysis. Sleep Med. 2018;43:100-108. doi:10.1016/j.sleep.2017.10.016

17. Kerley CP, Hutchinson K, Bolger K, McGowan A, Faul J, Cormican L. Serum Vitamin D is significantly inversely associated with disease severity in caucasian adults with obstructive sleep apnea syndrome. Sleep. 2016;39:293-300. doi:10.5665/sleep.5430

18. Tahar A, Zerdoumi F, Saidani M, Griene L, Koceir EA. Effects of oral vitamin $\mathrm{D}(3)$ supplementation in stage 3 chronic kidney disease subjects: insulin resistance syndrome and hormonal disturb interactions. Ann Biol Clin (Paris). 2018;76:313-325. doi:10.1684/ abc. 2018.1342
19. Puckrin R, Iqbal S, Zidulka A, Vasilevsky M, Barre P. Renoprotective effects of continuous positive airway pressure in chronic kidney disease patients with sleep apnea. Int Urol Nephrol. 2015;47:1839-1845. doi:10.1007/s11255-015-1113-y

20. Berry RB, Brooks R, Gamaldo CE et al. The AASM manual for the scoring of sleep and associated events: rules, terminology and technical specifications, version 2.6.0. American Academy of Sleep Medicine, Darien, Illinois; 2020. Available from: http://www. Aasmnet.Org. Accessed April 10, 2020.

21. Kapur VK, Auckley DH, Chowdhuri S, et al. Clinical practice guideline for diagnostic testing for adult obstructive sleep apnea: an American Academy of Sleep Medicine Clinical Practice Guideline. J Clin Sleep Med. 2017;13:479-504. doi:10.5664/jcsm.6506

22. Erden ES, Genc S, Motor S, et al. Investigation of serum bisphenol $\mathrm{A}$, vitamin $\mathrm{D}$, and parathyroid hormone levels in patients with obstructive sleep apnea syndrome. Endocrine. 2014;45:311-318. doi:10.1007/s12020-013-0022-z

23. Wang H, Chen W, Li D, et al. Vitamin D and chronic diseases. Aging Dis. 2017;8:346-353. doi:10.14336/AD.2016.1021

24. Archontogeorgis K, Nena E, Papanas N, et al. Vitamin D levels in middle-aged patients with obstructive sleep apnoea syndrome. Curr Vasc Pharmacol. 2018;16:289-297. doi:10.2174/ 1570161115666170529085708

25. Archontogeorgis K, Nena E, Papanas N, et al. Metabolic syndrome and vitamin D levels in patients with obstructive sleep apnea syndrome. Metab Syndr Relat Disord. 2018;16:190-196. doi:10.1089/met.2017.0181

26. Archontogeorgis K, Papanas N, Rizos EC, et al. Reduced serum Vitamin D levels are associated with insulin resistance in patients with obstructive sleep apnea syndrome. Medicina (Kaunas). 2019;55 (5):174. doi:10.3390/medicina55050174

27. Fan $\mathrm{Z}$, Cao B, Long $\mathrm{H}$, et al. Independent association of vitamin $\mathrm{D}$ and insulin resistance in obstructive sleep apnea. Ann Endocrinol (Paris). 2019;80:319-323. doi:10.1016/j.ando.2019.09.004

28. Wang W, Zhang J, Wang H, Wang X, Liu S. Vitamin D deficiency enhances insulin resistance by promoting inflammation in type 2 diabetes. Int J Clin Exp Pathol. 2019;12:1859-1867.

29. Archontogeorgis K, Nena E, Papanas N, Steiropoulos P. The role of vitamin D in obstructive sleep apnoea syndrome. Breathe (Sheff). 2018;14:206-215. doi:10.1183/20734735.000618

30. Mete T, Yalcin Y, Berker D, et al. Obstructive sleep apnea syndrome and its association with vitamin D deficiency. J Endocrinol Invest. 2013;36:681-685. doi:10.3275/8923

31. Yassa OY, Domac SF, Kenangil G. Serum Vitamin D status does not correlate with the severity of obstructive sleep apnea in male adults: a controlled study design with minimized factors influencing serum Vitamin D levels. Int J Vitam Nutr Res. 2019;1-7. doi:10.1024/03009831/a000539

32. Goswami U, Ensrud KE, Paudel ML, et al. Vitamin D concentrations and obstructive sleep apnea in a multicenter cohort of older males. Ann Am Thorac Soc. 2016;13:712-718. doi:10.1513/AnnalsATS.201507-440OC

33. Di Cesar DJ, Ploutz-Snyder R, Weinstock RS, Moses AM. Vitamin $\mathrm{D}$ deficiency is more common in type 2 than in type 1 diabetes. Diabetes Care. 2006;29:174. doi:10.2337/diacare.29.01.06.dc051876

34. Deleskog A, Hilding A, Brismar K, Hamsten A, Efendic S, Östenson CG. Low serum 25-hydroxyvitamin D level predicts progression to type 2 diabetes in individuals with prediabetes but not with normal glucose tolerance. Diabetologia. 2012;55:1668-1678.

35. Harris SS. Does vitamin D deficiency contribute to increased rates of cardiovascular disease and type 2 diabetes in African Americans? Am J Clin Nutr. 2011;93:1175s-8s. doi:10.3945/ajcn.110.003491

36. Boucher BJ. Hypovitaminosis D and risk of Type 2 diabetes in British South Asians. Diabet Med. 2006;23:336. doi:10.1111/j.14645491.2006.01825a.x 
37. Nsiah-Kumi PA, Erickson JM, Beals JL, et al. Vitamin $\mathrm{D}$ insufficiency is associated with diabetes risk in Native American children. Clin Pediatr (Phila). 2012;51:146-153. doi:10.1177/ 0009922811417290

38. Hussain Gilani SY, Bibi S, Siddiqui A, Ali Shah SR, Akram F, Rehman MU. Obesity and diabetes as determinants of vitamin D deficiency. J Ayub Med Coll Abbottabad. 2019;31:432-435.
39. Song Y, Wang L, Pittas AG, et al. Blood 25-hydroxy vitamin D levels and incident type 2 diabetes: a meta-analysis of prospective studies. Diabetes Care. 2013;36:1422-1428. doi:10.2337/dc12-0962

40. Bozkurt NC, Cakal E, Sahin M, Ozkaya EC, Firat H, Delibasi T. The relation of serum 25-hydroxyvitamin-D levels with severity of obstructive sleep apnea and glucose metabolism abnormalities. Endocrine. 2012;41:518-525. doi:10.1007/s12020-012-9595-1

\section{Publish your work in this journal}

Diabetes, Metabolic Syndrome and Obesity: Targets and Therapy is an international, peer-reviewed open-access journal committed to the rapid publication of the latest laboratory and clinical findings in the fields of diabetes, metabolic syndrome and obesity research. Original research, review, case reports, hypothesis formation, expert opinion and commentaries are all considered for publication. The manuscript management system is completely online and includes a very quick and fair peer-review system, which is all easy to use. Visit http://www.dovepress.com/testimonials.php to read real quotes from published authors. 